\title{
Pericardial diverticula misdiagnosed as pericardial cysts
}

\author{
Mary E. Money, MD, FACP, ${ }^{\mathrm{a}, \mathrm{b}}$ and Chong Park, MD, FACS, ${ }^{\mathrm{c}}$ Baltimore and Hagerstown, Md, and Jefferson \\ Borough, $\mathrm{Pa}$
}

Pericardial cysts, which result from complete closure of an embryonic pericardial defect, are believed to occur at a frequency of 1 in 100,000, based on a 1958 chest radiograph screening. ${ }^{1}$ Surgery is routinely not recommended, unless obvious compression of adjacent organs is demonstrated. However, $10 \%$ of all cysts may be instead a pericardial diverticulum with a persistent connection to the pericardial space, not apparent from radiologic studies, and identified only at surgery. ${ }^{2}$ These lesions may cause atypical symptoms that are relieved only after surgery, as demonstrated by this case report.

\section{CASE REPORT}

A 66-year-old retired biologist was diagnosed in 1984 with an "enlarged fat pad," by chest radiograph. Chest computed tomography (CT) scans revealed a right, cardiophrenic, $2.5-\mathrm{cm}$, soft-tissue cystic mass that had increased to $7.3 \mathrm{~cm}$ by 2008 (Figure 1). Additional studies included: standard cardiac exercise stress tests in 1989, 1993, and 1998 (all normal); exercise stress tests with images in 2001 and 2008 (all normal); cardiac catheterization in 2003 (negative for significant disease); echocardiograms in 2001, 2005, and 2008 (mild mitral insufficiency); 24hour Holter monitors in 1993 and 2001 (normal); cardiac magnetic resonance imaging in 2008; negative testing for pheochromocytoma in 2010; and a magnetic resonance imaging echocardiogram in 2012 (a $4.7 \times 3.6 \times 4.1 \mathrm{~cm}$ "pericardial cyst" without evidence of compression).

Intermittent, daytime, anterior chest symptoms, not affecting the patient's usual activities, were characterized as follows: nonradiating; unrelated to activity, inspiration, or position; duration from minutes to hours; and intensity of 2 to 5 on a scale of 0 to 10 . Different nocturnal symptoms occurred lasting 2-12 hours: over $90 \%$ of the episodes woke her from sleep: forceful heart pounding, intense anxiety, tachycardia,

\footnotetext{
From the Department of Internal Medicine, ${ }^{\mathrm{a}}$ University of Maryland School of Medicine, Baltimore; Department of Internal Medicine, ${ }^{\mathrm{b}}$ Meritus Medical Center, Hagerstown, Md; and Heart Institute at Jefferson Regional Medical Center, ${ }^{\mathrm{c}}$ Allegheny Health Network, Jefferson Borough, $\mathrm{Pa}$.

Disclosures: Authors have nothing to disclose with regard to commercial support.

Received for publication Nov 13, 2014; revisions received Dec 13, 2014; accepted for publication Jan 16, 2015; available ahead of print Feb 20, 2015.

Address for reprints: Mary E. Money, MD, FACP, Drs Waldman \& Money, PA, 354

Mill St, Hagerstown, MD 21740 (E-mail: dr.m.e.money@gmail.com).

J Thorac Cardiovasc Surg 2015;149:e103-7

0022-5223

Copyright (C) 2015 by The American Association for Thoracic Surgery. Published by Elsevier Inc. This is an open access article under the CC BY-NC-ND license (http:// creativecommons.org/licenses/by-nc-nd/4.0/).

http://dx.doi.org/10.1016/j.jtcvs.2015.01.032
}

inability to lie back down, mild chest discomfort sometimes, but without dyspnea, nausea, indigestion, flushing or diaphoresis. The episodes were not related to exercise, stress at work, the sudden death of her husband, eating, drinking, sexual activity, or urinating or bowel habits.

Frequency was not predictable, either for the vague daytime chest discomfort, or the nocturnal attacks, which both ranged from being weekly episodes, to years apart. Empiric treatment with beta-blockers for presumed supraventricular tachycardia decreased her heart rate, but did not lessen the other symptoms that accompanied the nocturnal attacks. Antianxiety medications did not abort them.

Symptoms began to increase in the spring of 2012. A portable electrocardiogram monitor recorded a normal sinus rhythm with a narrow complex during a night attack. Severe episodic fatigue attacks lasting $<2$ hours occurred, with a "heart-pounding sensation," unrelated to activity. While the patient was resting in a recliner, a family member noted a substantial neck-vein distention. However, by the time the patient saw her internist, symptoms had resolved, and the examination was normal.

Because of the positional symptoms, a connection between the "cyst" and the pericardial space was hypothesized, which would allow a shift of fluid from the lesion into the pericardium, possibly resulting in a localized transient pericardial effusion affecting the venous return to the right atrium. The patient was again referred to another thoracic surgeon, who did not attribute the symptoms to the "cyst" but agreed to perform surgery. On May 25, 2012, the patient underwent video-assisted thoracoscopic

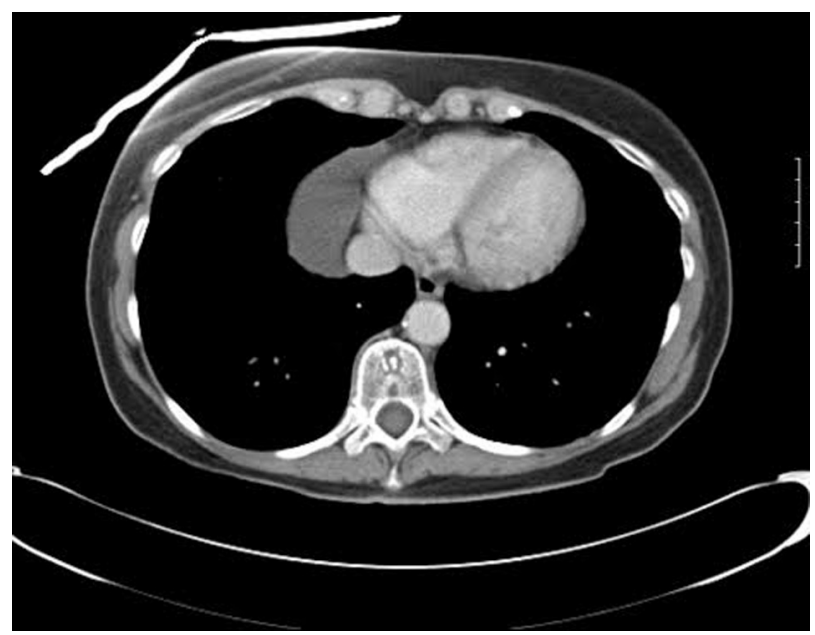

FIGURE 1. Chest computed tomography scan showing a right, cardiophrenic, 7.3-cm, soft-tissue, cystic mass. 
surgery to remove the "cyst." A diverticulum with a 4-to$5-\mathrm{mm}$ communication to the pericardium was found and removed. The patient has been asymptomatic since surgery.

\section{DISCUSSION}

As demonstrated by the preceding description, current radiologic studies, which fail to include this diagnosis, cannot differentiate a pericardial diverticulum from a cyst. A search of PubMed entries in the range of dates from 1946 to the present identified 36 cases of pericardial diverticula in English (Appendix E1 provides references describing the cases reviewed). Of these 36 cases, although symptoms were mentioned in only $24(66 \%), 16$ of the $24(66 \%)$ had clinical complaints, including 11 with chest discomfort. Eight $(33 \%)$ were asymptomatic. In addition, patients reported other complaints, identified in Appendix E1. After surgery, resolution of symptoms was reported in 13 of 17 cases including the case report $(76 \%)$. Pericardial diverticula may therefore cause atypical symptoms without compression, compared with pericardial cysts, which resolve after surgery.

Recent literature reports do not discuss the shift of fluid from the pericardial space to the diverticulum, or the reverse, which could contribute to the symptoms, but this shift has been clearly documented in numerous earlier cases. This shift was first reported in 1946, by Dr Mazer ${ }^{3}$ describing Dr Sweet's surgical report on a pericardial diverticulum:
... it was noticed that the fluid within the mass began to disappear, and, in squeezing it, all the fluid was expressed into the pericardial cavity. When the pressure was released, the action of the heart slowly pushed the fluid back into the sac, distending it once more about as much as when it was first seen. ... The communicating opening was a little more than $0.5 \mathrm{~cm}$ in diameter.

This case report, therefore, encourages a thoracic surgeon to include the possibility of diagnosis of a pericardial diverticulum when evaluating a patient with a symptomatic pericardial cyst, and supports the recommendation made previously by Akiba and colleagues ${ }^{4}$ “. ... that a symptomatic anterior mediastinal cyst be completely resected even if it does not appear large enough to compress the neighboring organs."

\section{References}

1. Le Roux BT. Pericardial coelomic cysts. Thorax. 1959;14:27-35.

2. Lyerly HK, Sabiston DC Jr. Primary neoplasms and cysts of the mediastinum. In: Fishman AP, ed. Pulmonary Diseases and Disorders. Vol 3, 2nd ed. New York: McGraw-Hill; 1988:2087-114.

3. Mazer ML. True pericardial diverticulum; report of a case, with safe operative removal. Am J Roentgenol Radium Ther. 1946;55:27-9.

4. Akiba T, Marushima H, Masubuchi M, Kobayashi S, Morikawa T. Small symptomatic pericardial diverticula treated by video-assisted thoracic surgical resection. Ann Thorac Cardiovasc Surg. 2009;15:123-5. 


\section{References}

E1. Mazer ML. True pericardial diverticulum; report of a case, with safe operative removal. Am J Roentgenol Radium Ther. 1946;55:27-9.

E2. Brown AL, Thomas SF. Hernia pericardii. Am J Surg. 1946;72:262-6.

E3. Bishop LF, Kirschner PA, Pessar TA. Diverticulum of the pericardium. Circulation. 1950;1:813-5.

E4. Brown RB, Dunn RG. Lymphogenous cysts of the mediastinum; cystic hygromas, pericardial cysts, and pericardial diverticulums. US Armed Forces Med J. 1951;2:1651-67.

E5. Halonen PI, Laitinen H. Pericardial diverticula. Ann Chir Gynaecol Fenn. 1953; 42:23-32.

E6. Perasalo O. On pericardial diverticula and their differential diagnosis. Acta Chir Scand. 1953;106:283-91.

E7. Ware GW, Conrad HA. Diverticula of the pericardium. Am J Surg. 1954;88: 918-21.

E8. Maier HC. Diverticulum of the pericardium with observations on mode of development. Circulation. 1957;16:1040-5.

E9. Fell SC, Schein CJ, Bloomberg AE, Rubinstein BM. Congenital diverticula of the pericardium. Ann Surg. 1959;149:117-25.

E10. Davis WC, German JD, Johnson NJ. Pericardial diverticulum causing pulmonary obstruction. Arch Surg. 1961;82:285-9.

E11. Kittredge RD, Finby N. Pericardial cysts and diverticula. Am J Roentgenol Radium Ther Nucl Med. 1967:99:668-73.

E12. Pader E, Kirschner PA. Pericardial diverticulum. Dis Chest. 1969;55:344-6.

E13. Waterbolk TW, Henkens CA, Remmert DG, van der Jagt EJ, Postmus PE. Pericardial diverticulum with unusual symptomatology. Eur Respir J. 1991;4: 232-4.

E14. Hirooka S, Ishiwara R. [A case of pericardial diverticulum with changing size]. Kyobu Geka. 1991;44:590-2. [abstract only; article in Japanese].
E15. Matushita Y, Taniguchi M, Niimi A, Suzuki K, Murayama T, Amitani R, et al [Two cases of pericardial diverticulum]. Nihon Kyobu Shikkan Gakkai Zasshi. 1992;30:1842-6. [abstract only; article in Japanese].

E16. Santoro MJ, Ford LJ, Chen YK, Solinger MR. Odynophagia caused by a pericardial diverticulum. Am J Gastroenterol. 1993;88:943-4.

E17. Wang FS, Wang QZ, Tian ZQ. [Diagnosis and management of pericardial cyst and diverticulum]. Zhonghua Wai Ke Za Zhi. 1994;32:240-1. [abstract only; article in Chinese].

E18. Yamamoto S, Mikami K, Sasaguri S, Hosoda Y, Danbara T, Suzuki K, et al. [A case report of pericardial diverticulum in the upper mediastinum: surgical treatment of two cases]. Kyobu Geka. 1995;48:242-5. [abstract only; article in Japanese].

E19. Ohno K, Yamasaki Y, Hatanaka N, Yamamoto S, Kuwata K. [Mediastinoscopic diagnosis and drainage of pericardial diverticulum-a case report]. Jpn J Thorac Cardiovasc Surg. 1998;46:496-8. [abstract only; article in Japanese].

E20. Takeda S, Miyoshi S, Minami M, Ohta M, Masaoka A, Matsuda H. Clinical spectrum of mediastinal cysts. Chest. 2003;124:125-32.

E21. Carretta A, Negri G, Pansera M, Melloni G, Zannini P. Thoracoscopic treatment of a pericardial diverticulum. Surg Endosc. 2003;17:158.

E22. Hanada S, Shirasawa B, Harada M, Ohnishi H, Tomozawa N, Hamano K. [Surgery for a pericardial diverticulum; report of a case]. Kyobu Geka. 2005;58 487-90. [abstract only; article in Japanese].

E23. Wei X, Liu L, Zhu X, Zhang Y, Duan L, Pan T. Pericardial diverticulum in the upper mediastinum. Ann Thorac Surg. 2009;87:e30.

E24. Akiba T, Marushima H, Masubuchi M, Kobayashi S, Morikawa T. Small symptomatic pericardial diverticula treated by video-assisted thoracic surgical resection. Ann Thorac Cardiovasc Surg. 2009;15:123-5.

E25. Guler A, Sahin MA, Kadan M, Erol G, Cingoz F, Tatar H. Incidental diagnosis of asymptomatic pericardial diverticulum. Tex Heart Inst J. 2011;38:206-7. 
APPENDIX E1. Pericardial diverticula reviewed

\begin{tabular}{|c|c|c|c|c|c|c|}
\hline Reference & $\begin{array}{l}\text { No. of } \\
\text { cases }\end{array}$ & Age (y) & Symptoms & $\begin{array}{l}\text { Study method: } \\
\text { Presurgical diagnosis }\end{array}$ & $\begin{array}{c}\text { Postsurgical } \\
\text { diagnosis }\end{array}$ & $\begin{array}{c}\text { Resolution of } \\
\text { symptoms } \\
\text { postsurgery Yes/No }\end{array}$ \\
\hline $\operatorname{Mazer}^{\mathrm{E} 1}(1946)$ & 1 & 55 & $\begin{array}{l}\text { Cough, epigastric distress, } \\
\text { mild nausea }\end{array}$ & $\begin{array}{l}\text { Chest radiograph: fat pad, } \\
\text { omental hernia, } \\
\text { mediastinal tumor }\end{array}$ & Pericardial diverticulum & $\begin{array}{l}\text { Yes, at time of } \\
\text { discharge, } 14 \mathrm{~d} \text { later }\end{array}$ \\
\hline Brown $^{\mathrm{E} 2}(1946)$ & 1 & 24 & Asymptomatic & $\begin{array}{l}\text { Chest radiograph: } \\
\text { mediastinal mass }\end{array}$ & Pericardial diverticulum & Not applicable \\
\hline Bishop $^{\mathrm{E} 3}(1950)$ & 1 & 26 & $\begin{array}{l}\text { Fatigue, vague left anterior } \\
\text { chest pain }\end{array}$ & $\begin{array}{l}\text { Chest radiograph: } \\
\text { pericardial cyst }\end{array}$ & Pericardial diverticulum & Unknown \\
\hline Brown $^{\mathrm{E} 4}(1951)$ & 1 & 27 & Asymptomatic & $\begin{array}{l}\text { Chest radiograph: pericardial } \\
\text { cyst }\end{array}$ & Pericardial diverticulum & Not applicable \\
\hline Halonen $^{\mathrm{E} 5}$ (1953) & 1 & 20 & $\begin{array}{l}\text { Chest pressure when } \\
\text { bending over }\end{array}$ & $\begin{array}{l}\text { Fluoroscopy: possible } \\
\text { pericardial diverticulum }\end{array}$ & Pericardial diverticulum & Unknown \\
\hline \multirow[t]{2}{*}{ Perasalo $^{\mathrm{E} 6}(1953)$} & 2 & 28 & $\begin{array}{l}\text { Chest pain intermittent for } 4 \text { y } \\
\text { Dyspnea } \\
\text { Dizziness }\end{array}$ & $\begin{array}{l}\text { Fluoroscopy: possible } \\
\text { pericardial diverticulum }\end{array}$ & Pericardial diverticulum & Yes \\
\hline & & 20 & Asymptomatic & $\begin{array}{l}\text { Fluoroscopy: possible } \\
\text { pericardial diverticulum }\end{array}$ & Pericardial diverticulum & Not applicable \\
\hline Ware $^{\mathrm{E} 7}(1954)$ & 1 & 47 & Asymptomatic & $\begin{array}{l}\text { Chest radiograph: } \\
\text { mediastinal mass }\end{array}$ & Pericardial diverticulum & Not applicable \\
\hline Maier $^{\mathrm{E} 8}$ (1957) & 1 & 29 & Occasional chest discomfort & $\begin{array}{l}\text { Chest radiographs: mass } \\
\text { at cardiac border }\end{array}$ & $\begin{array}{l}\text { Pericardial cyst and } \\
\text { diverticulum }\end{array}$ & Yes \\
\hline \multirow[t]{4}{*}{ Fell $^{\mathrm{E9}}$ (1959) } & 4 & 44 & Asymptomatic & $\begin{array}{l}\text { Chest radiograph: mediastinal } \\
\text { mass }\end{array}$ & Pericardial diverticulum & Not applicable \\
\hline & & 55 & $\begin{array}{l}\text { Nonexertional, intermittent, } \\
\text { anterior chest pain for } 2 \mathrm{wk}\end{array}$ & $\begin{array}{l}\text { Chest radiograph: rounded } \\
\text { density right paracardiac } \\
\text { region }\end{array}$ & Pericardial diverticulum & Yes \\
\hline & & 56 & Asymptomatic & $\begin{array}{l}\text { Chest radiograph: } \\
\text { mediastinal mass }\end{array}$ & Pericardial diverticulum & Not applicable \\
\hline & & 56 & Asymptomatic & mediastinal mass & Pericardial diverticulum & Not applicable \\
\hline $\operatorname{Davis}^{\mathrm{E} 10}(1961)$ & 1 & 10 & $\begin{array}{l}\text { Recurrent pulmonary } \\
\text { infections, precordial } \\
\text { chest pain, dyspnea }\end{array}$ & $\begin{array}{l}\text { Chest radiograph: pericardial } \\
\text { or bronchogenic cyst }\end{array}$ & Pericardial diverticulum & Yes \\
\hline $\begin{array}{l}\text { Kittredge }{ }^{\mathrm{E} 11} \\
(1967)\end{array}$ & 1 & 60 & Dyspnea & $\begin{array}{l}\text { Chest radiograph: } \\
\text { pericardial diverticulum }\end{array}$ & Pericardial diverticulum & Unknown \\
\hline Pader $^{\mathrm{E} 12}$ (1969) & 1 & 35 & Asymptomatic & $\begin{array}{l}\text { Chest radiograph: left } \\
\text { hilar mass }\end{array}$ & Pericardial diverticulum & Not applicable \\
\hline $\begin{array}{l}\text { Waterbolk }^{\mathrm{E} 13} \\
\text { (1991) }\end{array}$ & 1 & 37 & $\begin{array}{l}\text { Recurrent attacks of pleuritic } \\
\text { chest pain for } 3 \mathrm{mo} \text {, } \\
\text { duration }<24 \mathrm{~h}\end{array}$ & $\begin{array}{l}\text { CT scan and MRI: } \\
\text { pericardial cyst }\end{array}$ & Pericardial diverticulum & Yes \\
\hline Hirooka $^{\mathrm{E} 14}(1991)$ & 1 & 39 & Unknown & Pericardial diverticulum & Pericardial diverticulum & Unknown \\
\hline $\begin{array}{l}\text { Matushita }^{\mathrm{E} 15} \\
(1992)\end{array}$ & 2 & $\begin{array}{l}60 \\
20\end{array}$ & $\begin{array}{l}\text { Unknown } \\
\text { Unknown }\end{array}$ & $\begin{array}{l}\text { Pericardial diverticulum } \\
\text { Mediastinal cyst }\end{array}$ & $\begin{array}{l}\text { Pericardial } \\
\text { diverticula (both) }\end{array}$ & $\begin{array}{l}\text { Unknown } \\
\text { Unknown }\end{array}$ \\
\hline Santoro $^{\mathrm{E} 16}(1993)$ & 1 & 31 & Odynophagia & CT scan: mediastinal mass & Pericardial diverticulum & Yes \\
\hline Wang $^{\mathrm{E} 17}$ (1994) & 3 & NA & Unknown & Mediastinal masses & Pericardial diverticula & Unknown \\
\hline $\begin{array}{l}\text { Yamamoto }^{\mathrm{E} 18} \\
\text { (1995) }\end{array}$ & 2 & $\begin{array}{l}54 \\
43\end{array}$ & $\begin{array}{l}\text { Unknown } \\
\text { Unknown }\end{array}$ & $\begin{array}{l}\text { CT scan and MRI: upper } \\
\text { mediastinal cysts: possible } \\
\text { bronchogenic, lymphatic, } \\
\text { esophageal, or pericardial } \\
\text { cyst }\end{array}$ & $\begin{array}{l}\text { Pericardial diverticulum } \\
\text { Pericardial diverticulum }\end{array}$ & $\begin{array}{l}\text { Unknown } \\
\text { Unknown }\end{array}$ \\
\hline Ohno $^{\text {E19 }}$ (1998) & 1 & 38 & Unknown & $\begin{array}{l}\text { CT scan: pretracheal } \\
\text { lymph node }\end{array}$ & Pericardial diverticulum & Unknown \\
\hline
\end{tabular}




\begin{tabular}{|c|c|c|c|c|c|c|}
\hline Reference & $\begin{array}{l}\text { No. of } \\
\text { cases }\end{array}$ & Age (y) & Symptoms & $\begin{array}{c}\text { Study method: } \\
\text { Presurgical diagnosis }\end{array}$ & $\begin{array}{c}\text { Postsurgical } \\
\text { diagnosis }\end{array}$ & $\begin{array}{c}\text { Resolution of } \\
\text { symptoms } \\
\text { postsurgery Yes/No }\end{array}$ \\
\hline Takeda ${ }^{\mathrm{E} 20}(2003)$ & 3 & NA & Unknown (all 3) & $\begin{array}{l}\text { Chest radiograph and } \\
\text { CT scans } \\
\text { Mediastinal cysts (3) }\end{array}$ & $\begin{array}{l}\text { Pericardial } \\
\quad \text { diverticulum (3) }\end{array}$ & $\begin{array}{l}\text { Unknown } \\
\text { (All 3) }\end{array}$ \\
\hline Carretta $^{\mathrm{E} 21}$ (2003) & 1 & 35 & Recurrent chest pain & $\begin{array}{l}\text { Chest radiograph: varied size } \\
\text { MRI: cystic lesion partially } \\
\text { attached to pericardium }\end{array}$ & Pericardial diverticulum & Yes \\
\hline Hanada $^{\mathrm{E} 22}(2005)$ & 1 & 64 & $\begin{array}{l}\text { Cough } \\
\text { Dyspnea }\end{array}$ & CT scan: pericardial cyst & Pericardial diverticulum & Unknown \\
\hline Wei $X^{\mathrm{E} 23}$ (2009) & 1 & 51 & $\begin{array}{l}\text { 8-y history of recurrent } \\
\text { right-side chest pain }\end{array}$ & $\begin{array}{l}\text { CT scan and MRI: upper } \\
\text { mediastinal cyst: } \\
\text { intrathoracic thyroid or } \\
\text { pericardial cyst }\end{array}$ & Pericardial diverticulum & Yes \\
\hline \multirow[t]{2}{*}{ Akiba $^{\mathrm{E} 24}$ (2009) } & 2 & 75 & $\begin{array}{l}\text { Left parasternal chest } \\
\text { pain for } 5 \mathrm{mo}\end{array}$ & $\begin{array}{l}\text { Chest radiograph and CT } \\
\text { scan: anterior } \\
\text { mediastinal cyst }\end{array}$ & Pericardial diverticulum & Yes \\
\hline & & 55 & $\begin{array}{l}\text { Chest discomfort and } \\
\text { pain for } 3 \text { mo }\end{array}$ & $\begin{array}{l}\text { Chest radiograph negative } \\
\mathrm{CT} \text { and MRI: pericardial cyst }\end{array}$ & Pericardial diverticulum & Yes \\
\hline Guler $^{\mathrm{E} 25}$ (2011) & 1 & 76 & Dyspnea, palpitations & $\begin{array}{l}\text { CT: pericardial cyst; mitral } \\
\text { stenosis; atrial fibrillation }\end{array}$ & Pericardial diverticulum & Yes \\
\hline
\end{tabular}

$C T$, Computed tomography; $M R I$, magnetic resonance imaging; $N A$, not available. 